\title{
Liver metastasis and local recurrence of meningeal hemangiopericytoma: a case report
}

\author{
Aimaiti Yasen ${ }^{1,2,3 \#}$, Bo Ran ${ }^{1,2,3 \#}$, Tiemin Jiang ${ }^{1}$, Yusufukadier Maimaitinijiati ${ }^{1,2,3}$, Ruiqing Zhang ${ }^{1}$, \\ Qiang Guo ${ }^{1}$, Yingmei Shao ${ }^{1,2,3}$, Tuerganaili Aji ${ }^{1,2,3}$, Hao Wen $^{1,2,3}$ \\ ${ }^{1}$ Department of Liver Hydatid Disease, Digestive and Vascular Surgery Center, The First Affiliated Hospital of Xinjiang Medical University, Urumqi \\ 830011, China; ${ }^{2}$ State Key Laboratory on Pathogenesis, Prevention and Treatment of High Incidence Diseases in Central Asia, Xinjiang Medical \\ University, Urumqi 830011, China; ${ }^{3}$ Xinjiang Key Laboratory of Echinococcosis, The First Affiliated Hospital of Xinjiang Medical University, \\ Urumqi 830011, China \\ \#These authors contributed equally to this work. \\ Correspondence to: Tuerganaili Aji; Hao Wen. Department of Liver Hydatid Disease, Digestive and Vascular Surgery Center, The First Affiliated \\ Hospital of Xinjiang Medical University, Urumqi 830011, China Email: tuergan78@sina.com; Dr.wenhao@163.com.
}

\begin{abstract}
This report describes the clinical features, diagnostic and treatment modalities of a 62-yearold female patient with liver metastasis and local recurrence of meningeal hemangiopericytoma (HPC), who presented with the clinical manifestations of memory deterioration, visual reduction and abdominal mass for one month. Skull and abdominal computed tomography (CT), magnetic resonance imaging (MRI) and 18FFluorodeoxyglucose positron emission tomography (FDG-PET)-CT were used for pre-surgery identification. Lesions without FDG uptake and less tumoral viability was observed through FDG-PETCT, which was characteristic features of HPC before operation. The intraoperative specimens from the liver resection and the calvarium were pathologically examined for further confirming the diagnosis. The patient underwent liver resection and bilateral frontal cerebral convex and parafalx resection as well as intracranial pressure sensor implantation for liver metastasis and local recurrence of HPC respectively. The patient recovered well and no recurrence or distant metastasis was found after 2-year follow-up. Early diagnosis and long-term follow-up are crucial for patients with hepatic metastasis or local recurrence of HPC, and FDGPET-CT is recommended as an ideal imaging tool. Radical resection of HPC lesions is considered as an optimal treating approach.
\end{abstract}

Keywords: Meningeal hemangiopericytoma (HPC); metastasis; liver; recurrence; case report

Submitted Oct 17, 2019. Accepted for publication Nov 25, 2019.

doi: $10.21037 /$ tcr.2019.12.05

View this article at: http://dx.doi.org/10.21037/tcr.2019.12.05

\section{Introduction}

Meningeal hemangiopericytoma (HPC), originated from Zimmerman adventitial cells around the capillaries or posterior capillary venules, is a rare interlobar malignant tumor of central nervous system (CNS). Morphologically, adventitial cells are spindle and close to the capillary reticular fiber membrane, and they have obvious multidirectional differentiation abilities (1). Due to some similarities with meningiomas, including prone sites, rich blood supply and attachment to meningeal membrane,
HPC has been considered as a subgroup of meningioma for some decades (2). However, HPC has the characteristics of malignant tumors, and intraoperative and postoperative treatment methods are quite different from meningioma (3). Thus, it is of great significance to clarify the diagnosis of HPC before treatment as early as possible.

Unlike usual benign meningiomas, meningeal HPC has a high rate of local recurrence and distant metastasis. It was shown in a retrospective study that $75 \%$ patients had the possibility of HPC recurrence within five years after initial treatment. Besides, there occurred distant 


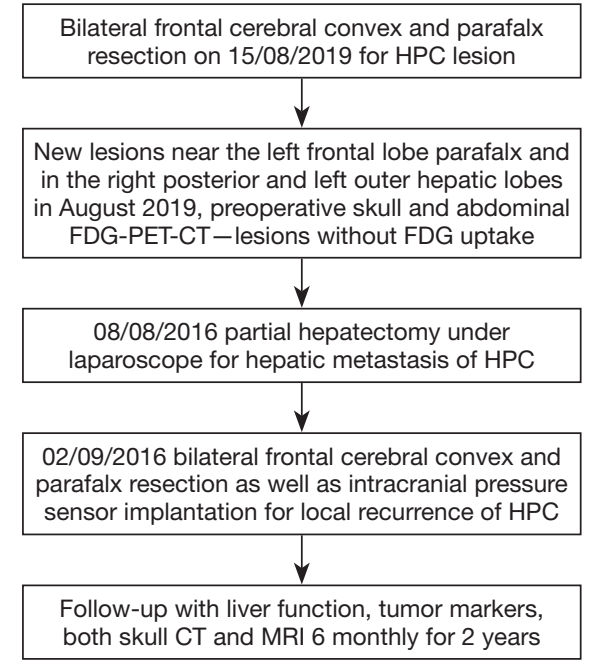

Figure 1 The timeline picture. The timeline picture presenting the related information and care of the patient.

metastasis in $17 \%$ HPC patients, majority of which was later than that of recurrence (4). Risk factors for the distant metastasis of HPC are as follows: malignant degree of HPC, operation times, radical extent of surgical resection, tumor viability etc. Traditional imaging techniques, such as X-ray, computed tomography (CT) and magnetic resonance imaging (MRI) may provide valuable information in diagnosis and differential diagnosis of metastatic as well as recurrent HPC before operation. Importantly, intra-operative biopsy or pathological examination for the surgical specimens are the golden standard for final diagnosis. Mena et al. demonstrated that the most common sites of HPC metastasis were lung (38\%), followed by liver $(13 \%)$, spleen and the lymph nodes $(11 \%)$ as well as the kidneys (8\%), which mainly metastasized to other distant organs through blood and the cerebrospinal fluid (5). The mean age of patients with HPC metastasis was 39 years and $75 \%$ of them were at the age of 38 to 55 . In addition, it has been accepted that most HPC may reoccur or metastasize 5 years later (6 months to 15 years) since the initial surgery $(6,7)$. This study was retrospectively analyzed patients from January 2015 to December 2018 in The First Affiliated Hospital of Xinjiang Medical University. In this study, we report a case involving a meningeal HPC patient with liver metastasis and local recurrence to summarize the clinical features, diagnostic and treatment modalities. We presented the following case in accordance with the CARE Guideline.

\section{Case presentation}

A 62-year-old female was referred to our institution for the evaluation and further management of her clinical symptoms. The timeline picture of the patient was as follows (Figure 1): 6 years' ago, the patient underwent bilateral frontal cerebral convex and parafalx resection for HPC. Now, the patient was presented with the clinical manifestations of memory deterioration, visual reduction and abdominal mass for one month and was admitted to our institution for further treatment. Physical examination showed no obvious abnormalities and the patient did not have special medical, family or psychosocial history. Preoperative skull and abdominal CT, MRI, ${ }^{18 \mathrm{~F}}$ Fluorodeoxyglucose Positron Emission Tomography (FDG-PET)-CT were used to estimate the recurrence and hepatic metastasis of HPC as well as assess the lesion size, location, parenchymal, vascular and biliary extension in the liver. Skull FDG-PET-CT showed high density lesion without FDG uptake and tumoral viability near the left frontal lobe parafalx (Figure 2A), demonstrating recurrence of HPC. In addition, both abdominal MRI and FDG-PETCT showed low density solid lesions without FDG uptake in the right posterior and left outer lobes of the liver (Figure $2 B, C, D)$. The radiological images suggested the possibility of hepatocellular carcinoma (HCC) or hepatocellular adenoma. Some results of laboratory tests were as follows: carbohydrate antigen (CA)19-9 was $59.05 \mathrm{ng} / \mathrm{mL}$; alpha-fetoprotein (AFP) $12.5 \mathrm{ng} / \mathrm{mL}$. Besides, the patient was also positive for hepatitis $\mathrm{B}$ surface antigen. Other clinical tests including full blood count-reactive protein, kidney and liver function tests were normal. Then, after careful assessment by a multidisciplinary team (MDT), including hepatobiliary surgeons, neurosurgeons, hepatologists, interventional therapists, radiologists and anesthesiologists, the patient underwent partial hepatectomy under laparoscope for hepatic metastasis of HPC, which lasted five hours and the intraoperative bleeding was $300 \mathrm{~mL}$. Due to relatively high intracranial pressure and postoperative malnutrition, the patient received antihypertensive and liver protection therapy as well as nutritional support treatment. 20 days later, bilateral frontal cerebral convex and parafalx resection as well as intracranial pressure sensor implantation was performed for local recurrence of HPC when the patient was able to bear some surgical trauma, 

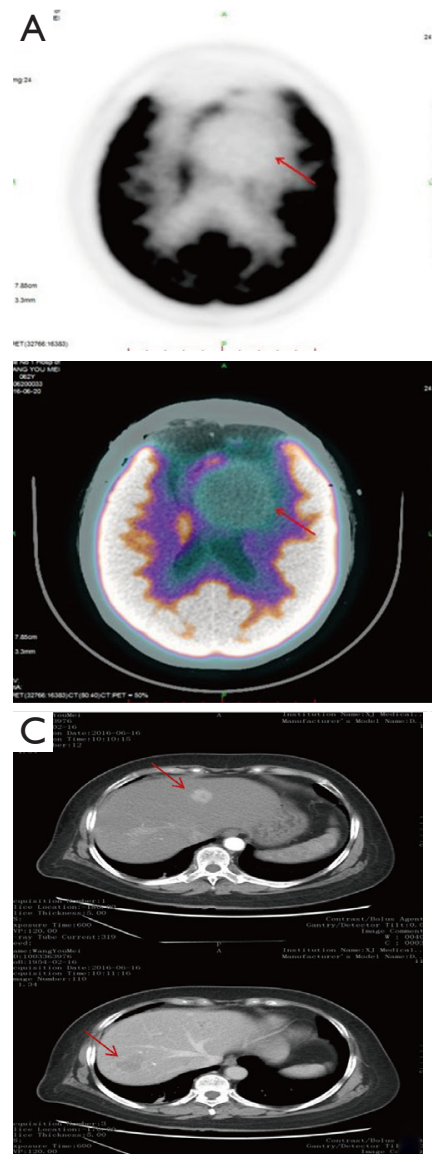
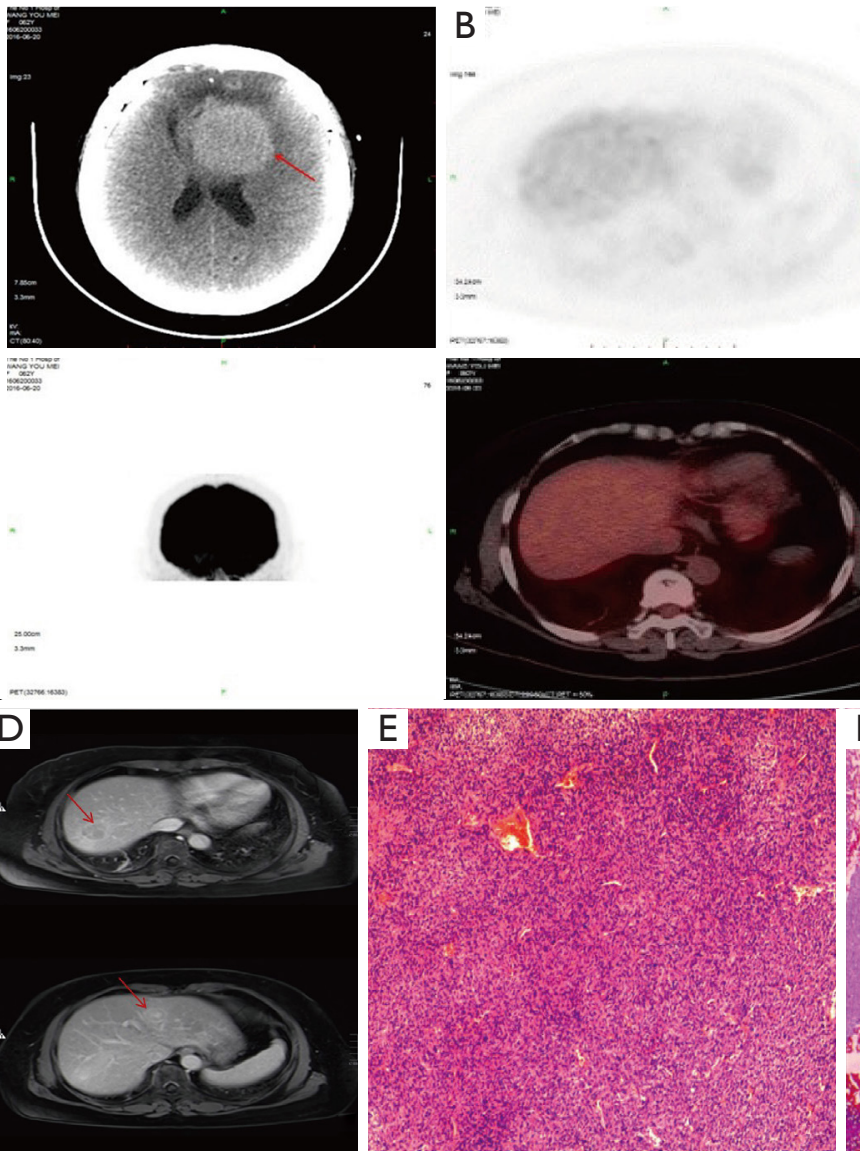
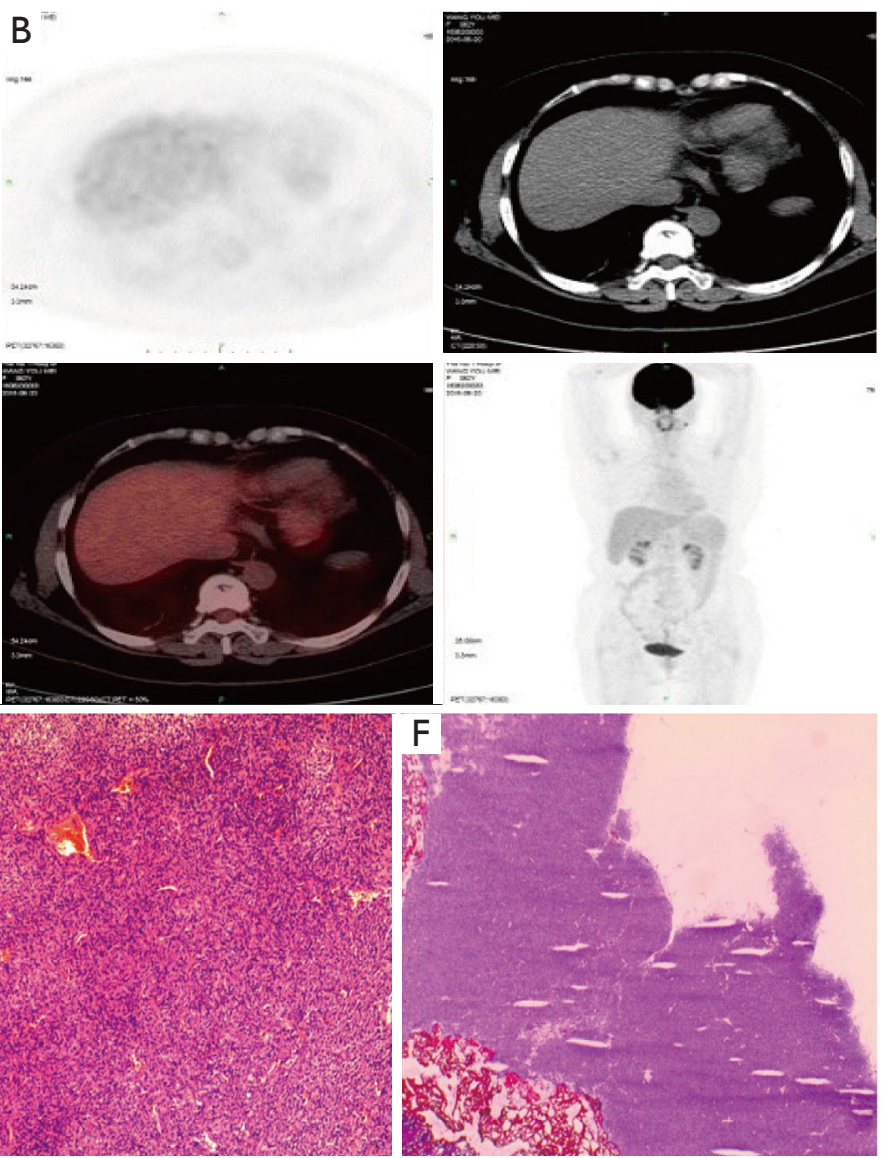

Figure 2 Representative imaging and postoperative pathological results. (A) Preoperative skull 18FDG-PET-CT, showing high density lesion without FDG uptake and less tumoral viability near the left frontal lobe parafalx (red arrows); (B,C,D) abdominal FDG-PET-CT and contrast-enhanced CT revealing low density solid lesions without FDG uptake in the right posterior and left outer lobes of the liver (red arrows); (E,F) hematoxylin eosin staining indicating the spindle-like tumor cells with moderate pleomorphism, oval nuclei and a moderate amount of cytoplasm (20x magnification). FDG-PET-CT, 18FFluorodeoxyglucose positron emission tomography computed tomography.

which lasted six hours and intraoperative bleeding was $800 \mathrm{~mL}$. All intraoperative specimens from liver resection and the calvarium were sent for rapid frozen section examination to verify the negative margins. Surgical specimens were examined for further confirmation of the diagnosis through bematoxylin eosin (H\&E) staining (Figure 2E,F). Histologically, the metastatic tumors in the liver and the recurrent tumors in the calvarium showed homogeneous features of HPC tumor cells with abundant cytoplasm, oval nuclei and moderate pleomorphism, which was also positive for vimentin, CD34 and vascular smooth muscle actin, and negative for cytokeratin (CK)8, CK18, CK19, AFP, A103,S100, CD99, desmin, epithelial membrane antigen (EMA) and glial fibrillary acidic protein (GFAP), further confirming the diagnosis. According to the
2016 World Health Organization (WHO) classification of tumors of CNS (8), the tumor belonged to WHO grade II. The patient recovered well and was successfully discharged 10 days after operation. And finally, in the 2-year followup, the patient was completely adherent and tolerable to the above intervention performed and there were not evident adverse or unanticipated events after treatment. The laboratory tests of liver function, some tumor markers during the follow-up were as follows: alanine transaminase (ALT) $20 \mathrm{U} / \mathrm{L}$, aspartate transaminase (AST) $22 \mathrm{U} / \mathrm{L}$, ALB $45 \mathrm{~g} / \mathrm{L}$, total bilirubin (TDIL) $5.23 \mu \mathrm{mol} / \mathrm{L}$, AFP $2.45 \mathrm{ng} / \mathrm{mL}$, carcino-embryonic antigen (CEA) $2.15 \mathrm{~g} / \mathrm{L}$, CA19-9 $12.45 \mathrm{ng} / \mathrm{ml}$. Besides, both skull CT and MRI (Figure 3) showed that there occurred some basic postoperative changes and the intracranial pressure was at 

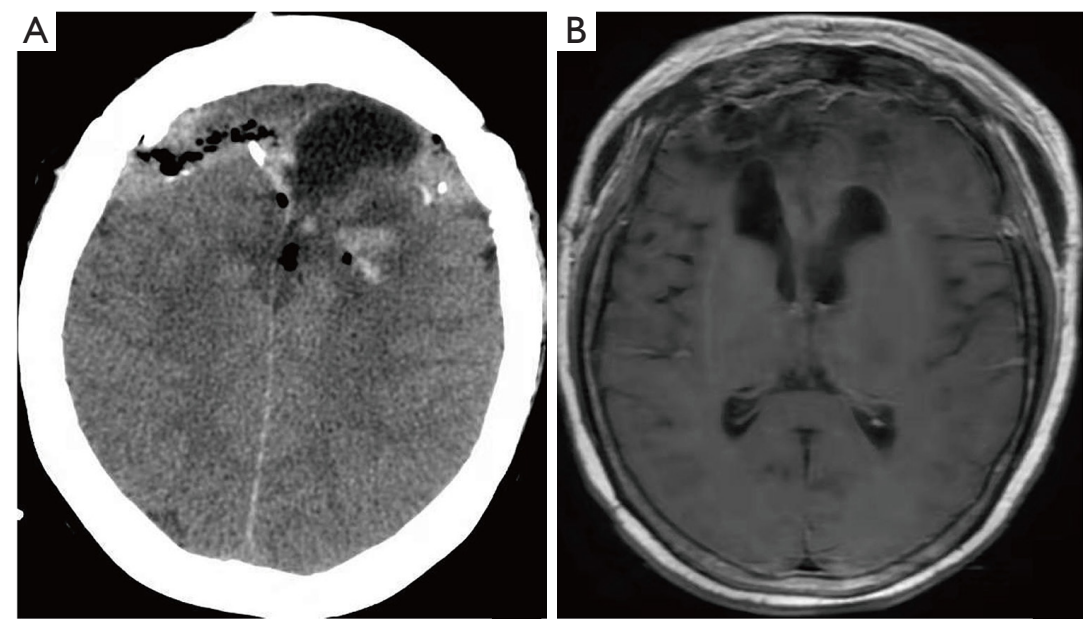

Figure 3 Typical postoperative imaging photos in 2-year follow-up. (A) Skull CT demonstrating basic postoperative changes in the bilateral frontal; (B) skull MRI also revealing no special abnormality only with some changes after surgery. CT, computed tomography; MRI, magnetic resonance imaging.

the normal level, suggesting no signs of local recurrence or distant metastasis of HPC.

\section{Discussion}

Mainly originated from mesenchymal tissues, HPC belongs to a malignant tumor, majority of which occurs in the CNS as the primary site. HPC accounts for approximately $2 \%$ of all meningeal tumors and $0.4 \%$ primary CNS tumors $(8,9)$. For decades, there were no distinct boundaries between meningeal HPC and typical meningioma due to some similarities of tumor location and histological features. However, according to WHO classification of CNS tumors, HPC was considered an independent stomal tumor in the CNS. Compared with benign meningioma, HPC tends to occur at a younger age and slightly more often in men than in women (10). Most importantly, HPC exhibits more aggressive biological behavior associated with high recurrence rate and frequent distant metastasis. Thus, it is suggested that patients should be diagnosed precisely and timely before initial treatment and closely followed up after treatment. In this study, we mainly described one patient with metastatic and recurrent HPC, who underwent surgery for HPC six years ago, to summarize the clinicopathological features and therapeutic methods.

When it comes to pre-operative initial diagnosis of HPC, imaging may provide some evidence. Through skull CT scan, it can be found that there may appear irregular lobulated shape with clear boundary in meningeal HPC.
In addition, most CT scan destiny in the primary lesions is slightly higher than that of the normal parenchyma (11). MRI may also demonstrate equal or low signal intensity on T1WI, high or mixed signal intensity on T2WI. On enhanced scan, lesion enhancement is obvious, and meningeal sign of mouse tail is not found (12). The above features can distinguish HPC from typical meningeal tumors. If there exits calcification in the lesions, the possibility of HPC may generally be excluded. In this study, skull FDG-PET-CT showed high density lesion without FDG uptake and less tumoral viability near the left frontal lobe parafalx, suggesting recurrence of HPC. Clinically, meningeal HPC is characterized by delayed metastasis, and liver is one of the most common sites. Hepatic mesenchymal tumors, primary or metastatic, are rare and not representative radiographically. Preoperative radiological results always demonstrate the possibility of HCC or hepatic hemangioma (7). On enhanced scan, both HCC and HPC lesions are enhanced. Moreover, in this study, the patient was known to be the carrier for hepatitis B, but she was not cirrhotic. The serum AFP level was almost within the normal level; thus, it is difficult to make precise diagnosis. Thankfully, abdominal FDG-PET-CT showed low density solid lesions with low FDG uptake, which is consistent with previous studies and may be characteristic features of metastatic HPC in the liver and can also distinguish from HCC (13).

Pathological examination serves as the golden standard for the diagnosis of HPC. spindle cells with moderate 
cellularity and round to oval nuclei are typically seen in HPC patients. Morphologically, HPC tumor cells are composed of medium size fusiform or polyhedral cells with oval nucleus and deep chromatin staining. In addition, there also appear plenty of typical horn-like blood vessels, and it is difficult to find normal meningioma structures, such as vortex-like structures and psammoma body in HPC lesions (14). HPC tumors are also rich in reticular fibers, some of which have anaplastic manifestations. Altogether, these above microscopic features may indicate the possibility of HPC. Immunohistochemically, vimentin, CD34, smooth muscle actin and desmin are highly expressed in HPC, while HPC is negative for EMA expression (10), which not only distinguish HPC from benign meningiomas, but also further confirm the diagnosis. H\&E staining of intraoperative specimens from liver resection and calvarium revealed spindle-like tumor cells with moderate pleomorphism, oval nuclei and a moderate amount of cytoplasm. Besides, tumor cells were positive for vimentin, CD34 and vascular smooth muscle actin, verifying the hepatic metastasis and local recurrence of HPC.

Many modalities, including surgical resection, radiotherapy, palliative therapy with irradiation or anti-angiogenic agents are considered to be promising therapeutic options for the treatment of HPC (15-17). By contrast, chemotherapy has been regarded as a disappointing therapy for this tumor (18). Except for the above treatment modalities, liver transplantation has also been reported as an optional candidate for the treatment of hepatic metastasis of HPC (19). The patient received partial hepatectomy, bilateral frontal cerebral convex and parafalx resection as well as intracranial pressure sensor implantation for the hepatic metastasis and recurrence of HPC respectively, and she did not occur any sign of recurrence or distant metastasis after operation during 2-year followup period. Taken together, the patient had representative imaging results of high-density lesion without FDG uptake for both hepatic metastasis and local recurrence of HPC, which may provide diagnostic reference for HPC. More importantly, the patient recovered well without evident adverse or unanticipated events after treatment, demonstrating that surgeons may follow the surgical methods performed in this study when encountering similar cases. However, this report has the following limitations: (I) due to the limited sample size with one case, the study only offer some recommendations for surgeons in the clinical settings. Thus, multi-institutional or multi-regional studies were further needed; (II) longer follow-up time is necessary for the final confirmation of distant metastasis or local recurrence of HPC.

\section{Conclusions}

To sum up, in spite of its rarity in clinical settings, HPC represents malignant tumor with high recurrence and metastasis rate. Thus, early diagnosis and long-term followup is crucial, and FDG-PET-CT is recommended as an ideal imaging tool. Radical resection of HPC lesions is still considered as an optimal treating approach for patients with hepatic metastasis or local recurrence of HPC.

\section{Acknowledgments}

The authors would like to acknowledge the technical support from Department of Liver Hydatid Disease, Digestive and Vascular Surgery Center, The First Affiliated Hospital of Xinjiang Medical University.

Funding: This study was supported by the General Program of National Natural Science Foundation of China (Grant No. 81660108) and the General Program of National Natural Science Foundation of Xinjiang Uyghur Autonomous Region (Grant No. 2016D01C274).

\section{Footnote}

Conflicts of Interest: All authors have completed the ICMJE uniform disclosure form (available at http://dx.doi. org/10.21037/tcr.2019.12.05). The authors have no conflicts of interest to declare.

Ethical Statement: The authors are accountable for all aspects of the work in ensuring that questions related to the accuracy or integrity of any part of the work are appropriately investigated and resolved. Design of this study and management of the patient were in accordance with the Helsinki Declaration (as revised in 2013). Written and signed informed consent was obtained from the patient or her legal custodies to publish the case. Publication of the patient data in the report were approved by the Ethical Committees of the First Affiliated Hospital of Xinjiang Medical University (Approval No: 201580731-3). Information revealing the subject's identity was avoided.

Open Access Statement: This is an Open Access article distributed in accordance with the Creative Commons Attribution-NonCommercial-NoDerivs 4.0 International 
License (CC BY-NC-ND 4.0), which permits the noncommercial replication and distribution of the article with the strict proviso that no changes or edits are made and the original work is properly cited (including links to both the formal publication through the relevant DOI and the license). See: https://creativecommons.org/licenses/by-nc-nd/4.0/.

\section{References}

1. Vogels RJ, Vlenterie M, Versleijen-Jonkers YM, et al. Solitary fibrous tumor-clinicopathologic, immunohistochemical and molecular analysis of 28 cases. Diagn Pathol 2014;9:224.

2. Righi V, Tugnoli V, Mucci A, et al. MRS study of meningeal hemangiopericytoma and edema: a comparison with meningothelial-meningioma. Oncol Rep 2012;28:1461-67.

3. Liu L, Yin B, Geng DY, et al. Comparison of ADC values of intra-cranial hemangiopericytomas and angiomatous and anaplastic meningiomas. J Neuroradiol 2014;41:188-94.

4. Nonake M, Kohmura E, Hirata M, et al. Metastatic Meningeal hemangiopericytoma of thoracic spine. Clin Neurol Neurosurg 1998;100:228-30.

5. Mena H, Ribas JL, Pezeshkpour GH, et al. Hemangiopericytoma of the central nervous system; a review of 94 cases. Hum Pathol 1991;22:84-91.

6. Schiariti M, Goetz P, El-Maghraby H, et al. Hemangiopericytoma: long-term outcome revisited. Clinical article. J Neurosurg 2011;114:747-55.

7. Lo RC, Suriawinata AA, Rubin BP. Liver metastasis of meningeal hemangiopericytoma: a study of 5 cases. Clin Mol Hepatol 2016;22:188-91.

8. Louis DN, Perry A, Reifenberger G, et al. The 2016 World Health Organization Classification of Tumors of the Central Nervous System: a summary. Acta Neuropathol 2016;131:803-20.

9. Mark W, Roy OW. Tumors of the central nervous system (AFIP atlas of tumor pathology, series 4. Fascicle 7).

Cite this article as: Yasen A, Ran B, Jiang T, Maimaitinijiati Y, Zhang R, Guo Q, Shao Y, Aji T, Wen H. Liver metastasis and local recurrence of meningeal hemangiopericytoma: a case report. Transl Cancer Res 2020;9(2):1278-1283. doi: 10.21037/ tcr.2019.12.05
Neuropathol Appl Neurobiol 2008;34:473-4.

10. Chang IW, Lin JW, Wu YT. The status of MGMT protein expression is a prognostic factor for meningeal hemangiopericytoma: a clinicopathologic and immunohistochemical study of 12 cases at a single institution. J Neurooncol 2011;105:563-72.

11. Dufour H, Métellus P, Fuentes S, et al. Meningeal hemangiopericytoma: a retrospective study of 21 patients with special review of postoperative external radiotherapy. Neurosurgery 2001;48:756-62; discussion 762-3.

12. Mama N, Ben Abdallah A, Hasni I, et al. MR imaging of intracranial hemangiopericytomas. J Neuroradiol 2014;41:296-306.

13. Chan WS, Zhang J, Khong PL. 18F-FDG-PETCT imaging findings of recurrent intracranial haemangiopericytoma with distant metastases. Br J Radiol 2010;83:e172-4.

14. Kim JH, Jung HW, Kim YS, et al. Meningeal hemangiopericytomas: long-term outcome and biological behavior. Surg Neurol 2003;59:47-53; discussion 53-4.

15. Hoshi M, Araki N, Naka N, et al. Bone metastasis of intracranial meningeal hemangiopericytoma. Int J Clin Oncol 2005;10:208-13.

16. Lee SJ, Kim ST, Park SH, et al. Successful use of pazopanib for treatment of refractory metastatic hemangiopericytoma. Clin Sarcoma Res 2014;4:13.

17. Park MS, Patel SR, Ludwig JA, et al. Activity of temozolomide and bevacizumab in the treatment of locally advanced, recurrent, and metastatic hemangiopericytoma and malignant solitary fibrous tumor. Cancer 2011;117:4939-47.

18. Staples JJ, Robinson RA, Wen BC, et al. Hemangiopericytoma-the role of radiotherapy. Int J Radiat Oncol Biol Phys 1990;19:445-51.

19. Urata K, Ikegami T, Nakazawa Y, et al. Living-donor liver transplantation for hepatic metastasis from meningeal hemangiopericytoma: A Case Report. Transplant Proc 2015;47:2274-7. 